\title{
Global Analysis of a Planetary Gear Train
}

\author{
Tongjie Li ${ }^{1,2}$ and Rupeng $\mathrm{Zhu}^{1}$ \\ ${ }^{1}$ College of Mechanical and Electrical Engineering, Nanjing University of Aeronautics and Astronautics, Nanjing 210016, China \\ ${ }^{2}$ College of Engineering, Anhui Science \& Technology University, Fengyang 233100, China
}

Correspondence should be addressed to Tongjie Li; litongjie2000@163.com

Received 16 May 2013; Accepted 22 July 2013; Published 16 February 2014

Academic Editor: Valder Steffen

Copyright (C) 2014 T. Li and R. Zhu. This is an open access article distributed under the Creative Commons Attribution License, which permits unrestricted use, distribution, and reproduction in any medium, provided the original work is properly cited.

\begin{abstract}
By using the Poincaré-like cell-to-cell mapping method and shooting method, the global characteristics of a planetary gear train are studied based on the torsional vibration model with errors of transmission, time-varying meshing stiffness, and multiple gear backlashes. The study results reveal that the planetary with a certain set of parameters has four coexisting periodic orbits, which are P-1, P-2, P-4, and P-8, respectively. P-1 and P-2 motions are not of long-term stability, P-8 motion is of local stability, and P-4 motion is of global stability. Shooting method does not have the capacity of searching coexisting periodic orbits in a global scope, and it is easy to omit some periodic orbits which are far away from the main gropes of periodic orbits.
\end{abstract}

\section{Introduction}

Because of the existence of some nonlinear factors, such as multiple gear backlashes and time-varying mesh stiffness, planetary gear train is an inherent strongly nonlinear dynamical system. Generally speaking, a nonlinear dynamical system with a certain set of parameters may have several periodic orbits [1], and different orbits may have different domains of attraction [2]. Making certain of all coexisting periodic orbits and their domains of attraction, designers of a planetary gear train can forecast the motion state of the system and the scope of disturbance that the motion state can bear.

Over the past three decades, the dynamics of planetary transmissions have drawn much attention, but almost all of the published studies on this field focused only on their linear vibration property [3-6]; the literature about nonlinear dynamics of a planetary transmission system is still limited $[7,8]$. As to global analysis of a planetary gear train, we can not find a study on this aspect in the existing literature.

Some scholars $[9,10]$ made development research about a method called cell-to-cell mapping method, which has demonstrated their great potential and efficiency to study the global characteristics of a general nonlinear dynamical system. In aspect of application of cell-to-cell mapping method to engineering, Liu and Chen [11] studied the global dynamic characteristics of a rotor-bearing nonlinear dynamical system by using Poincaré-like cell-to-cell mapping method. Polchai and Hsu [12] studied domains of stability of a synchronous generator by a cell-to-cell method.

The objective of this paper is to study all of the coexisting periodic solutions and their domains of attraction of a planetary gear train based on a nonlinear torsional vibration model with multiple backlashes, time-varying mesh stiffness, and static transmission errors.

\section{Dynamic Model of a Planetary Gear Train}

In this study, the planetary gear system concerned is a singlestage $2 \mathrm{~K}-\mathrm{H}$ type planetary gear set as shown in Figure 1 . Some detailed modeling progress has been published in [8] by the author of this paper. The model consists of a sun gear $\mathrm{S}$, a ring gear $\mathrm{R}$, planet gears $\mathrm{P}$, and a carrier $\mathrm{C}$. Translational degrees of every components in this model are eliminated, and only rotational motions of every component bodies are considered. Rotational motions of the ring, carrier gear, sun gear, and planet gears are denoted by $\theta_{h}, h=r, c, s, p_{1}, p_{2}$, $\ldots, p_{N}$, where $N$ indicates the number of planet gears. In this paper, $\theta_{r}=0$ since the ring gear is fixed on gearbox. The character $r_{b h}, h=s, r, p_{1}, p_{2}, \ldots, p_{N}, c$, stands for the base circle radius for the sun, ring, planets, and the radius of the circle passing through the planet centers for the carrier, respectively. 


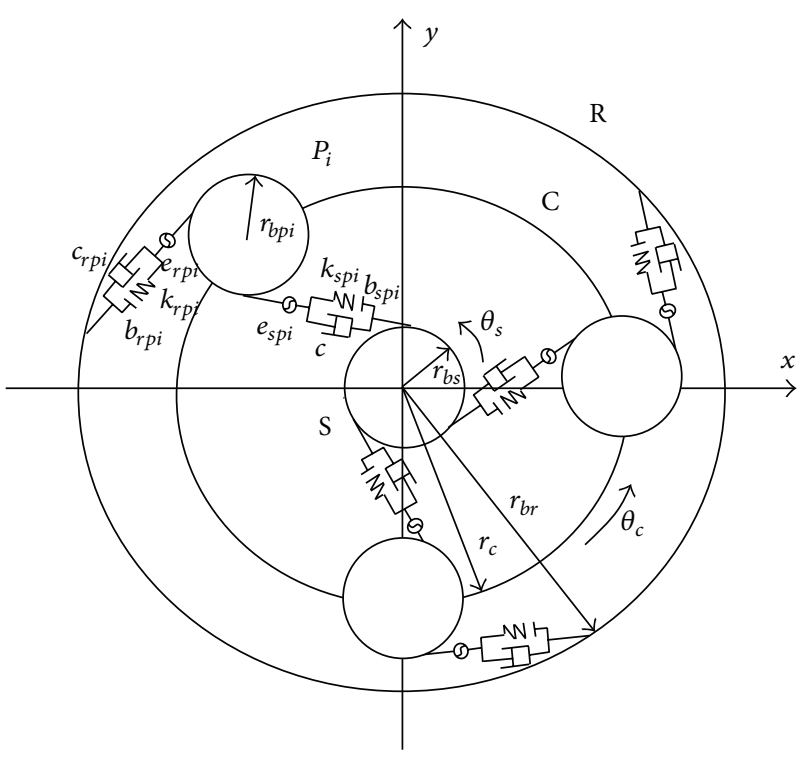

FIGURE 1: Nonlinear dynamical model of planetary gear train.

$k_{s p i}, c_{s p i}, b_{s p i}$, and $e_{s p i}$ denote the backlash, time-varying mesh stiffness, viscous damping and the static transmission error of the sun gear-planet gear- $i$ pair; $k_{r p i}, c_{r p i}, b_{r p i}$, and $e_{r p i}$ denote the corresponding terms in the ring gear-planet gear- $i$ pair. This nomenclature also applies to other physical parameters of the corresponding meshing pairs.

2.1. Time-Varying Mesh Stiffness. For spur gears, rectangular waves are often used to approximate mesh stiffness alternating between $d$ and $d+1$ pairs of teeth in contact [13]. Expanding rectangular wavesin Fourier series and taking the first harmonic term of the system's mesh frequency, the timevarying mesh stiffness in corresponding mesh pair can be assumed as

$$
\begin{aligned}
& k_{s p i}(t)=k_{m s p i}+k_{a s p i} \sin \left(\omega t+\varphi_{s p i}\right), \\
& k_{r p i}(t)=k_{m r p i}+k_{a r p i} \sin \left(\omega t+\varphi_{r p i}\right),
\end{aligned}
$$

where $k_{m s p i}$ and $k_{m r p i}$ are mean values of the stiffness in the corresponding meshing pair, $k_{a s p i}$ and $k_{\text {arpi }}$ are time-varying amplitude of the stiffness in the corresponding meshing pair, $\varphi_{s p i}$ and $\varphi_{r p i}$ are initial phases of the stiffness in the corresponding meshing pair, and $\omega$ is the system's mesh frequency.

2.2. Static Transmission Errors. The static transmission error is one of the primary excitations of planetary gear train's vibration and noise. According to the study of Parker [14], the static transmission errors in the corresponding mesh pair $e_{s p i}(t)$ and $e_{r p i}(t)$ can be expressed as

$$
\begin{gathered}
e_{s p i}(t)=e_{a s p i} \sin \left(\omega t+\beta_{s p i}\right), \\
e_{r p i}(t)=e_{a r p i} \sin \left(\omega t+\beta_{r p i}+\gamma_{s r}\right),
\end{gathered}
$$

where $e_{a s p i}$ and $e_{r s p i}$ are time-varying amplitude of the static transmission error in the corresponding meshing pair, $\beta_{s p i}$ and $\beta_{r p i}$ are initial phases of the static transmission error in the corresponding meshing pair, and $\gamma_{s r}$ denotes the phase between the sun-planet and ring-planet mesh error for a given planet.

2.3. Nonlinear Displacement Function about Backlash. According to the study of Sun and $\mathrm{Hu}$ [7], the nonlinear displacement function about backlash is as follows:

$$
f(X, b)= \begin{cases}X-b & X>b \\ 0 & |X| \leq b \\ X+b & X<-b\end{cases}
$$

where $X$ denotes the relative gear mesh displacement and $b$ denotes half backlash in the corresponding mesh pair.

2.4. Equations of Motion. In order to eliminate rigid body motion and simplify equations of the system, $N_{1}$ (in this paper, $N_{1}=N+1$ ) dimension equivalent displacements coordinate $X_{s p i}$ and $X_{s c}$ are introduced according to the model shown in Figure 1:

$$
\begin{gathered}
X_{s p i}=\theta_{s} r_{b s}-\theta_{p i} r_{b p i}-\theta_{c} r_{c} \cos \alpha-e_{s p i}(t), \\
X_{s c}=\theta_{s} r_{b s}-2 \theta_{c} r_{c} \cos \alpha,
\end{gathered}
$$

where $\alpha$ is the pressure angle; in this paper all the pressure angles of different gear are of the same value.

The dimensionless torsional motion equations of the planetary gear system, which are shown as (5), can be established by using the Lagrange principle and equivalent displacements coordinate (4):

$$
\begin{aligned}
\ddot{\bar{X}}_{s p i} & +\frac{1}{\omega_{n}^{2} m_{e q 1}} \\
& \times \sum_{i=1}^{3}\left[k_{m s p i}+k_{a s p i} \sin \left(\tau \Omega+\beta_{s i}\right)\right] f\left(\bar{X}_{s p i}, \bar{b}_{s p i}\right) \\
& +\frac{1}{\omega_{n} m_{e q 1}} \sum_{i=1}^{3} c_{s p i} \dot{\bar{X}}_{s p i}+\frac{1}{\omega_{n}^{2} m_{p i}} \\
& \times\left[k_{m s p i}+k_{a s p i} \sin \left(\tau \Omega+\beta_{s i}\right)\right] f\left(\bar{X}_{s p i}, \bar{b}_{s p i}\right) \\
& +\frac{1}{\omega_{n} m_{p i}} c_{s p i} \dot{\bar{X}}_{s p i}-\frac{1}{\omega_{n}^{2} m_{p i}} \\
& \times\left[k_{m r p i}+k_{a r p i} \sin \left(\tau \Omega+\beta_{s i}\right)\right]
\end{aligned}
$$




$$
\begin{aligned}
& \times f\left(\bar{X}_{s c}-\bar{X}_{s p i}-\bar{e}_{s p i}-\bar{e}_{r p i}, \bar{b}_{r p i}\right) \\
& -\frac{1}{\omega_{n} m_{p i}} c_{r p i}\left(\dot{\bar{X}}_{s c}-\dot{\bar{X}}_{s p i}-\dot{\bar{e}}_{s p i}-\dot{\bar{e}}_{r p i}\right) \\
& +\frac{1}{\omega_{n}^{2} m_{c}} \sum_{i=1}^{3}\left[k_{m r p i}+k_{a r p i} \sin \left(\tau \Omega+\beta_{s i}\right)\right] \\
& \times f\left(\bar{X}_{s c}-\bar{X}_{s p i}-\bar{e}_{s p i}-\bar{e}_{r p i}, \bar{b}_{r p i}\right) \\
& +\frac{1}{\omega_{n} m_{c}} \sum_{i=1}^{3} c_{r p i}\left(\dot{\bar{X}}_{s c}-\dot{\bar{X}}_{s p i}-\dot{\bar{e}}_{s p i}-\dot{\bar{e}}_{r p i}\right) \\
= & \frac{F_{D}}{\left(m_{s} b_{c} \omega_{n}^{2}\right)}+\frac{F_{D}}{\left(m_{s} b_{c} \omega_{n}^{2}\right)}+\frac{\left.2 F_{n}^{2}\right)}{\left(m_{c} b_{c} \omega_{n}^{2}\right)} . \ddot{\bar{e}}_{s p i}(\tau) \\
\ddot{\bar{X}}_{s c}+ & \frac{1}{\omega_{n}^{2} m_{e q 2}} \sum_{i=1}^{3}\left[k_{m s p i}+k_{a s p i} \sin \left(\tau \Omega+\beta_{s i}\right)\right] \\
& \times f\left(\bar{X}_{s c}-\bar{X}_{s p i}-\bar{e}_{s p i}-\bar{e}_{r p i}, \bar{b}_{r p i}\right) \\
& \times f\left(\bar{X}_{s p i}, \bar{b}_{s p i}\right)+\frac{1}{\omega_{n} m_{e q 2}} \\
& \times \sum_{i=1}^{3} c_{r p i}\left(\dot{\bar{X}}_{s c}-\dot{\bar{X}}_{s p i}-\dot{\bar{e}}_{s p i}-\dot{\bar{e}}_{r p i}\right) \\
& \times \sum_{i=1}^{3} \dot{\bar{X}}_{s p i} \frac{2}{\omega_{n} m_{c}}
\end{aligned}
$$

Some dimensionless parameters are introduced in the process of deducing (5):

$$
\Omega=\omega / \omega_{n}, \tau=\omega_{n} t, \omega_{n}=\sqrt{k_{m s p 1} / m_{e q 1}}, m_{e q 1}=
$$
$m_{c} m_{s} /\left(m_{c}+m_{s}\right), m_{e q 2}=m_{c} m_{s} /\left(m_{c}+2 m_{s}\right), m_{s}=J_{s} / r_{b s}^{2}$, $m_{p i}=J_{p i} / r_{b p i}^{2}, m_{c}=\left(J_{c}+\sum_{i=1}^{3} m_{p i} r_{c}^{2}\right) / r_{b c}^{2}, F_{D}=T_{D} / r_{b s}$, $F_{L}=T_{L} / r_{b s}, X=\bar{X} b_{c}, \dot{X}=\dot{\bar{X}} b_{c} \omega_{n}, \ddot{X}=\ddot{\bar{X}} b_{c} \omega_{n}^{2}, b=b_{c} \bar{b}$, $e=b_{c} \bar{e}$, where $b_{c}$ is a character length, $J_{s}, J_{p_{i}}, J_{c}$ are the inertia of the sun gear, $i$ th planet gear, and the carrier.

\section{Introduction about Poincaré-Like Cell-to- Cell Mapping Method}

The cell-to-cell mapping method, which is introduced by Hsu [9], is an efficient method that can be used to study the global characteristics of strong nonlinear systems. According to cellto-cell mapping method, one can divide a large state space of a nonlinear system into finitely many small state spaces, which is called cell space, and study coexisting periodic orbits and their domains of attraction can be turned into the question about mapping relations of finitely many small cells in cell spaces.

The main disadvantage of cell-to-cell mapping method is that the computation quantity is too large to study the global characteristics of high dimensional nonlinear dynamical system. Aiming at this defect, Levitas et al. [10] put forward to Poincaré-like cell-to-cell mapping method and bring down the computation quantity of global analysis for a nonlinear dynamical system.

The state equations of $n$ dimension nonautonomous dynamical system with periodicity can be written as follows:

$$
\dot{x}=f(t, x, \mu)=f(t+T, x, \mu), \quad(t, x, \mu) \in \mathbf{R} \times \mathbf{R}^{n} \times \mathbf{R},
$$

where $T$ is the period of the dynamical system and $\mu$ is a parameter of the system.

Using Poincaré cell-to-cell mapping method, (6) can be changed into a discrete point-to-point mapping in a selected Poincaré section $\sum$, which is represented as

$$
x^{(k+1)}=p\left(x^{(k)}, \mu\right), \quad k \in Z,
$$

where $p: \sum \rightarrow \sum$.

Searching the periodic solutions $x(t)=x(t+k T, \mu), k \in Z$ is changed into calculating the fixed points $x^{*}$ in the point-topoint mapping (7), which satisfies the following formula

$$
x^{*}=p^{k}\left(x^{*}, \mu\right), \quad k \in Z .
$$

Using point-to-point mapping (7), global analysis of the dynamical system (6) in the whole state space is simplified to calculating the fixed points and their domains of attraction in the selected Poincaré section $\sum$. Because the dimension of the Poincare section $\sum$ is less than the whole state space, the computation quantity to study the global characteristics of a high dimensional dynamical system in $\sum$ can be reduced significantly.

One can reduce the computation quantity significantly again by constructing a cell-to-cell mapping in the Poincaré section $\sum$, which is called Poincaré-like cell-to-cell mapping:

$$
z(m+l)=p^{l}(z(m), \mu), \quad l \in Z,
$$

where $Z(m)$ is a cell vector of integer elements which shows the position of the cell in state space and $p^{l}$ means executing the cell-to-cell mapping $l$ times. For a given cell $z(m)$, one can find its central point $x^{d}(m)$ as the start point and carry out point-to-point map (7) $l$ times to get its image point $x^{d}(m+l)$. Judging which cell the map point $x^{d}(m+l)$ is located in, one can get the image cell $z(m+1)$. It is easy to get the coexisting periodic cells and their domains of attraction by judging the relations of cells in cell apace.

All of the cells wich the fixed points $x^{*}$ are located in make up the periodic cells which reflect the periodic solutions $x(t)$ of dynamical system (6). In specific operation of Poincarélike cell-to-cell mapping, let mapping times $l$ be a large integer; for example, $l=50$; the calculated periodic cells will be of long-term stability. 


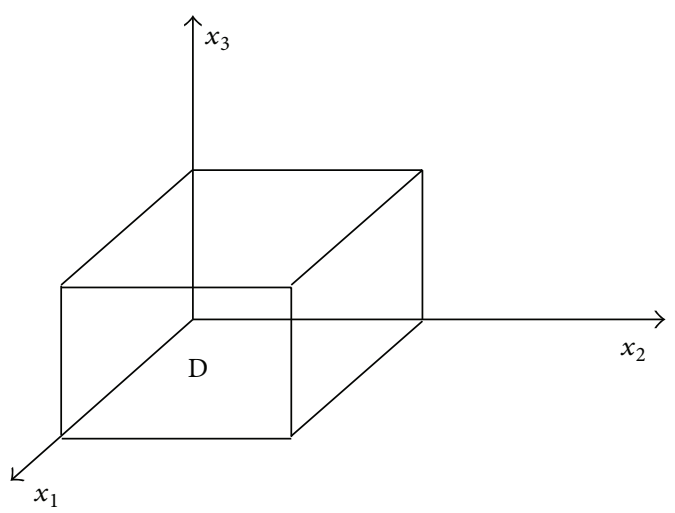

Figure 2: The studied scope $\mathrm{D}$ on Poincaré section $\sum=$ $\left\{\left(x_{1}, x_{2}, x_{3}, x_{4}\right) \mid x_{4}=0\right\}$.

All of the cells which are attracted into a set of periodic cells constitute the domain of attraction of these periodic cells, and the magnitude of the domain of attraction reflects the degree of stability of the corresponding periodic solutions $x(t)$.

\section{Global Analysis of Planetary Gear Train}

4.1. Coexisting Periodic Orbits with Long-Term Stability. The main parameters of the planetary gear system showed in Figure 1 are as follows $m=3 \mathrm{~mm}, \alpha=20^{\circ}, e_{\text {aspi }}=e_{\text {arpi }}=$ $10 \mu \mathrm{m}, z_{s}=15, z_{p i}=24, z_{r}=63, b_{c}=10 \mu \mathrm{m}, b_{s p i}=b_{r p i}=$ $50 \mu \mathrm{m}, N=3, k_{m s p i}=0.8256 \times 10^{9} \mathrm{~N} / \mathrm{m}, k_{m r p i}=1.06 \times$ $10^{9} \mathrm{~N} / \mathrm{m}, \beta_{\mathrm{si}}=\beta_{r i}=0, \varphi_{s p i}=\varphi_{r p i}=0, k_{a s p i}=0.3 \times 10^{9} \mathrm{~N} / \mathrm{m}$, and $k_{\text {arpi }}=0.3 \times 10^{9} \mathrm{~N} / \mathrm{m}$.

Assuming all of the external meshing pairs are of the same mesh stiffness, static transmission error and backlash, and internal meshing pairs have similar assumptions, and (5) will become differential equation which is of 2 degrees of freedom about the relative displacements. Let sequence of solution vector $x(t)$ of the state equations corresponding to (5) be $x(t)=\left(x_{1}, x_{2}, x_{3}, x_{4}\right)=\left(\bar{X}_{s p i}(t), \dot{\bar{X}}_{s p i}(t), \bar{X}_{s c}(t), \dot{\bar{X}}_{s c}(t)\right)$.

Because $x_{2}$ and $x_{4}$ denote velocity of the relative displacements $x_{1}$ and $x_{3}$, respectively, all of the periodic orbits must pass through the following two hyperplane sections: $x_{2}=0$ and $x_{4}=0$. In this paper, hyperplane section $\sum=$ $\left\{\left(x_{1}, x_{2}, x_{3}, x_{4}\right) \mid x_{4}=0\right\}$ is regarded as Poincaré section. The studied scope D in Poincaré section $\sum$ is a rectangular block showed as Figure 2, which is formed by the following 3 coordinate axes, $x_{1}, x_{2}, x_{3}$. Global analysis in Poincaré section $\sum$ can bring down the work of calculation immensely.

Considering the position of periodic fixed points which can be solved by using shooting method [15], the studied scope D in Poincaré section $\sum$ can be determined as

$$
\begin{aligned}
& -25 \leq x_{1} \leq 25 \\
& -10 \leq x_{2} \leq 20 \\
& -10 \leq x_{3} \leq 50 .
\end{aligned}
$$

A cell space can be constructed by discretizing the studied scope $\mathrm{D}$ in $\sum$ into $50 \times 30 \times 60$ cells. In order to express periodic

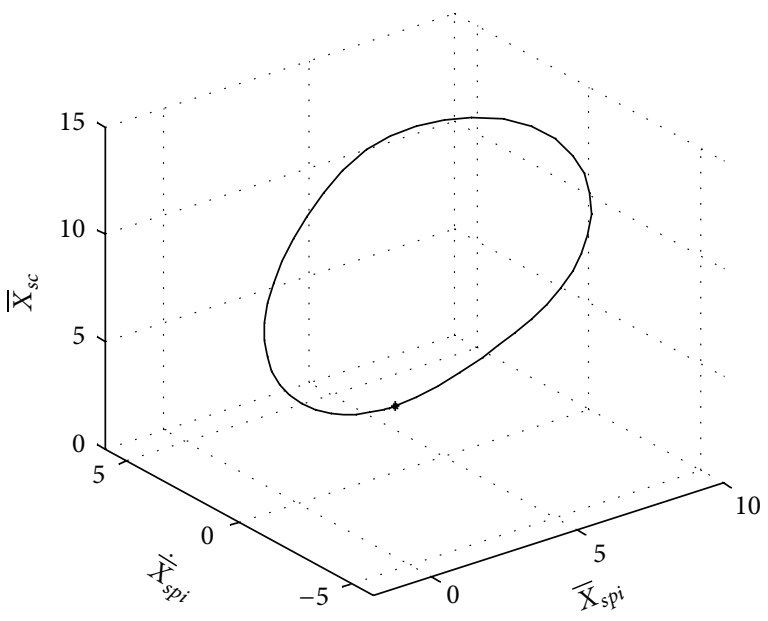

FIgURE 3: P-1 fixed points and periodic orbits projected into D.

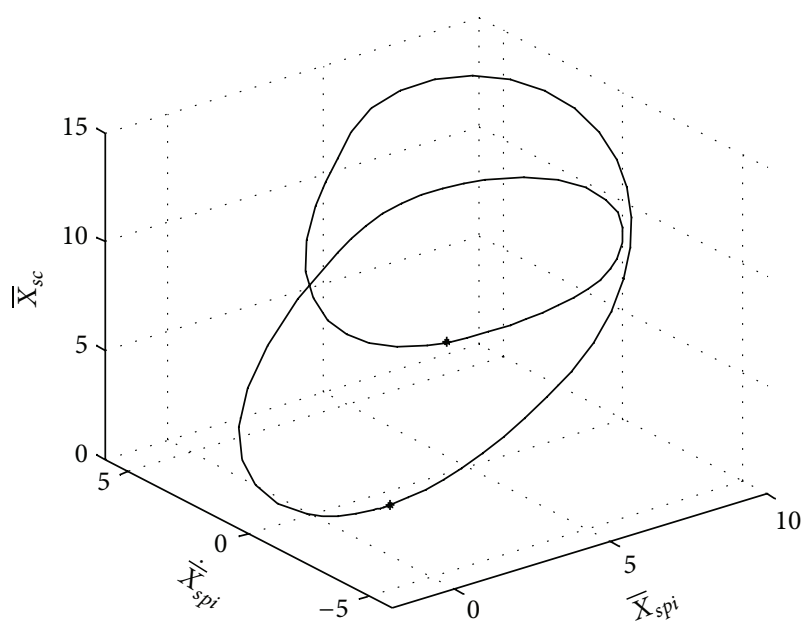

FIGURE 4: P-2 fixed points and periodic orbits projected into D.

solution of the system that is of $m$ times period of the system conveniently, the simplified expression P-m is introduced in this paper. By using shooting method, 3 coexisting periodic fixed points are searched in $\mathrm{D}$, which are $\mathrm{P}-1$ periodic fixed points $p_{1}=[1.2736,-2.7122,5.5372], \mathrm{P}-2$ periodic fixed points including $P_{21}=[0.3119,-2.9195,1.9834]$ and $P_{22}=[2.2329,-2.7524,8.5388], \mathrm{P}-4$ periodic fixed points including $P_{41}=[0.0584,-2.3862,2.5963], P_{42}=$ $[0.9718,-2.8886,7.4437], P_{43}=[1.0232,-3.3769,2.3746]$, and $P_{44}=[2.9127,-2.6107,8.8167]$, respectively.

Starting from P-1 periodic fixed points, integrate the state equation corresponding to (5) in a period of the system by using the fixed-step fourth order Runge-Kutta method; the phase trajectory of the P-1 periodic orbit which is projected into $\mathrm{D}$ can be obtained and shown as Figure 3. By using the same method, P-2 and P-4 periodic orbits projected into $\mathrm{D}$ can be obtained and shown as Figures 4 and 5, respectively. Asterisks in these figures denote the positions of corresponding periodic fixed points. 


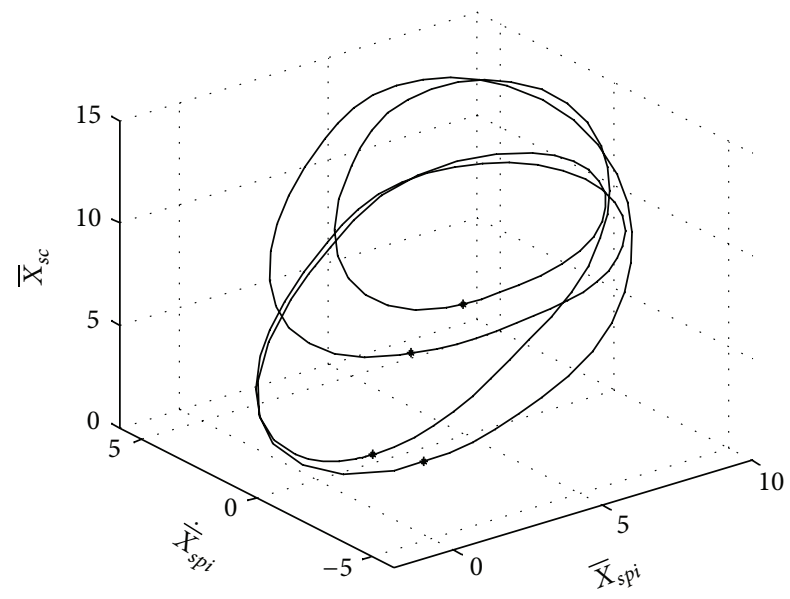

FIGURE 5: P-4 fixed points and periodic orbits projected into D.

Through the verification by using direct numerical integration method, one can find that only P-4 motion of these three coexisting periodic orbits is of the long-term stability. Namely, as to P-1 and P-2, there are no domains of attraction.

By using Poincaré-like cell-to-cell mapping method, two kinds of coexisting periodic cells which are of long-term stability are found. One is P-4 periodic cell; another is P-4 periodic cell. By contrast, P-4 periodic cell found by Poincarélike cell-to-cell mapping method and $\mathrm{P}-4$ periodic fixed point found by shooting method stand for the same periodic orbit. P-8 periodic cells comprise the following points in cell space: $P_{81}=[-9.8296,3.4673,-8.4349], P_{82}=[-10.0250,3.9632$, $-2.4987], P_{83}=[-9.3758,3.9118,-6.4858], P_{84}=[-10.1171$, $3.3448,-4.5905], P_{85}=[-9.8296,3.4673,-8.4349], P_{86}=$ $[-10.0250,3.9632,-2.4987], \quad P_{87}=[-9.3758,3.9118$, $-6.4858], P_{88}=[-10.1171,3.3448,-4.5905]$, respectively.

The phase trajectory of the $\mathrm{P}-8$ periodic orbit which is projected into $\mathrm{D}$ can be obtained and shown as Figure 6 , and the asterisks in Figure 6 denote the positions of corresponding periodic cells.

Two conclusions can be gotten from the above study.

(1) With the given set of parameters, planetary gear set has two coexisting periodic orbits with long-term stability; one is $\mathrm{P}-4$ periodic motion, and another is $\mathrm{P}-8$ periodic motion.

(2) The shooting method does not have the capacity of searching coexisting periodic orbits in a global scope.

Namely, the shooting method only finds some coexisting periodic orbits which are close to each other and easy to omit some periodic orbits which are far away from the main periodic orbit groups.

4.2. Domain of Attraction of Coexisting Periodic Orbits with Long-Term Stability. By using Poincaré-like cell-to-cell mapping method, domain of attraction of $\mathrm{P}-8$ periodic orbits with long-term stability can be calculated and shown as Figure 7. The marks " $\square$ " denote the position of P-8 periodic cell, the marks "." denote cells of attraction of P-8 periodic orbits,

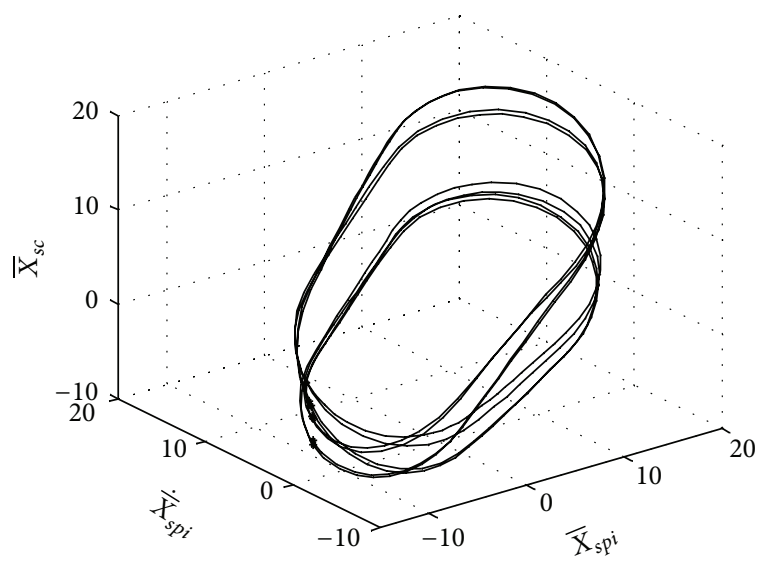

FIGURE 6: P-8 fixed points and periodic orbits projected into D.

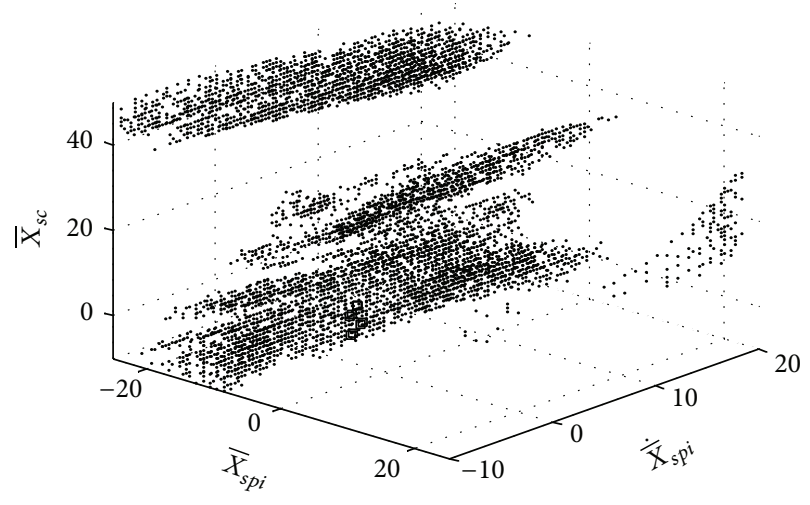

FIgURE 7: Domain of attraction of P-8 periodic orbit.

and all of the cells constitute the domain of attraction of P8 periodic orbits.

The degree of stability of $\mathrm{P}-8$ periodic orbits can be measured by the scope of domain of attraction; namely, a bigger scope of domain of attraction shows that the corresponding periodic orbits are more stable. If the disturbance of $\mathrm{P}-8$ periodic orbit is limited in its domain of attraction, the motion will keep stable.

There are 5470 cells in the domain of attraction of P-8 periodic orbits showed in Figure 6, and $6.08 \%$ of the whole cell space in $\mathrm{D}$ belonged to this domain of attraction. The domain of attraction showed in Figure 7 is composed of three subspaces. Periodic cells of P-8 are full of the three subspaces, but it is not continuous among each of the subspaces.

Some conclusions about stability of $\mathrm{P}-8$ periodic orbit can be gotten from the above discussions. Because the domain of attraction is full of the occupied cell spaces, $\mathrm{P}-8$ periodic orbit is of local stability; namely, if disturbance of this orbit is limited in its domain of attraction, the orbit will keep stable, and if disturbance increases to be out of its domain of attraction, the orbit will lose its stability and turn into other coexisting periodic orbits.

Figure 8 is domain of attraction of P- 4 periodic orbit. Except for the cell space which is occupied by domain of 


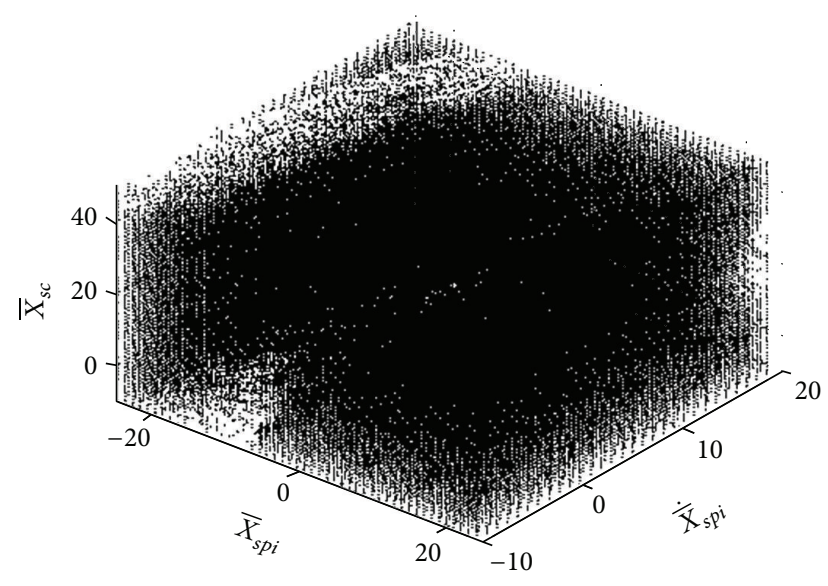

FIGURE 8: The domain of attraction of P-4 motion.

attraction of P-8, the domain of attraction of P-4 is almost full of the whole cell space. There are 84530 cells attracted into P-4 periodic orbit; namely, $93.92 \%$ of cells in the whole cell space belong to domain of attraction of P-4 periodic orbit. It is obvious that $\mathrm{P}-4$ periodic orbit is of global stability.

\section{Conclusions}

(1) By using Poincare-like cell-to-cell method and shooting method, the global characteristics of a planetary gear set are studied based on a nonlinear torsional dynamical model with gear backlashes and timevarying mesh stiffness.

(2) With the given set of parameters, planetary gear set has two coexisting periodic orbits with long-term stability; one is $\mathrm{P}-4$ periodic motion, and another is $\mathrm{P}-8$ periodic motion. The $\mathrm{P}-4$ periodic motion is of global stability and the $\mathrm{P}-8$ periodic motion is of local stability.

(3) The shooting method is not of the capacity of searching coexisting periodic orbits in a global scope. Namely, the shooting method can only find some coexisting periodic orbits which are close to each other and easy to omit some periodic orbits which are far away from main periodic orbit groups.

\section{Conflict of Interests}

The authors declare that there is no conflict of interests regarding the publication of this paper.

\section{Acknowledgments}

The authors gratefully acknowledge the support received through the National Nature Science Foundation of China (50775108) and the General Natural Science Foundation of Anhui Science \& Technology University (ZRC2013382).

\section{References}

[1] J. Li and S. Zhang, "Cell-mapping computation method for nonsmooth dynamical systems," Acta Mechanica Solida Sinica, vol. 28, no. 1, pp. 93-96, 2007 (Chinese).

[2] C. S. Hsu and J. X. Xue, "The global analysis of higher order nonlinear dynamical systems and the application of cell-to-cell mapping method," Applied Mathematics and Mechanics, vol. 11, pp. 1035-1044, 1985.

[3] J. Lin and R. G. Parker, "Analytical characterization of the unique properties of planetary gear free vibration," Journal of Vibration and Acoustics, Transactions of the ASME, vol. 121, no. 3, pp. 316-321, 1999.

[4] R. G. Parker, V. Agashe, and S. M. Vijayakar, "Dynamic response of a planetary gear system Using a finite element/contact mechanics model," Journal of Mechanical Design, Transactions of the ASME, vol. 122, no. 3, pp. 304-310, 2000.

[5] A. Saada and P. Velex, "Extended model for the analysis of the dynamic behavior of planetary trains," Journal of Mechanical Design, Transactions of the ASME, vol. 117, pp. 241-247, 1995.

[6] A. Kahraman, "Planetary gear train dynamics," Journal of Mechanical Design, Transactions of the ASME, vol. 116, no. 3, pp. 713-720, 1994.

[7] T. Sun and H. Hu, "Nonlinear dynamics of a planetary gear system with multiple clearances," Mechanism and Machine Theory, vol. 38, no. 12, pp. 1371-1390, 2003.

[8] T. J. Li and R. P. Zhu, "Study on stability about motion state and bifurcation properties of planetary gear train," Journal of Central South University of Technology, vol. 6, pp. 1543-1547, 2012.

[9] C. S. Hsu, "A theory of cell-to-cell mapping for dynamical systems," Journal of Applied Mechanics, Transactions ASME, vol. 47, no. 4, pp. 931-939, 1980.

[10] J. Levitas, T. Weller, and J. Singer, "Poincaré-like simple cell mapping for non-linear dynamical systems," Journal of Sound and Vibration, vol. 176, no. 5, pp. 641-662, 1994.

[11] H. Liu and S. T. Chen, "A new method PCM for analyzing global dynamic characteristic of nonlinear system and its application to rotor bearing system," Chinese Journal of Applied Mechanics, no. 3, pp. 7-12, 1995 (Chinese).

[12] A. Polchai and C. S. Hsu, "Domains of stability of a synchronous generator by a cell mapping approach," International Journal of Control, vol. 41, no. 5, pp. 1253-1271, 1985.

[13] J. Lin and R. G. Parker, "Planetary gear parametric instability caused by mesh stiffness variation," Journal of Sound and Vibration, vol. 249, no. 1, pp. 129-145, 2002.

[14] R. G. Parker, "Physical explanation for the effectiveness of planet phasing to suppress planetary gear vibration," Journal of Sound and Vibration, vol. 236, no. 4, pp. 561-573, 2000.

[15] Y.-G. Luo, Z.-H. Ren, H. Ma, T. Yu, and B.-C. Wen, "Stability of periodic motion on the rotor-bearing system with coupling faults of crack and rub-impact," Journal of Mechanical Science and Technology, vol. 21, no. 6, pp. 860-864, 2007. 

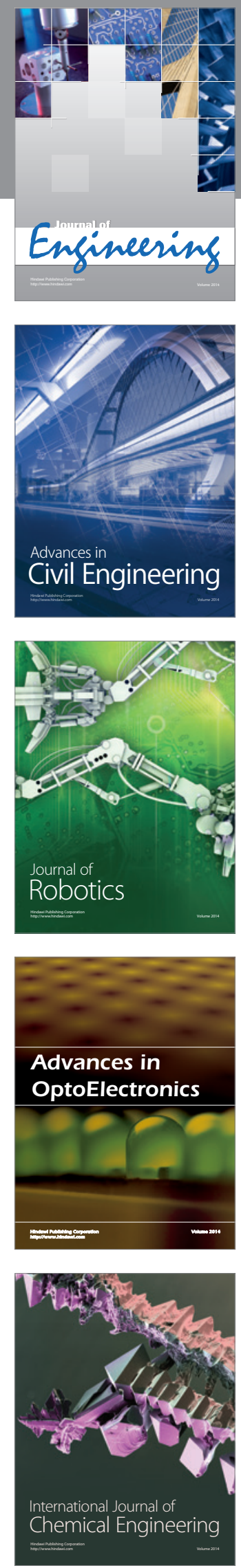

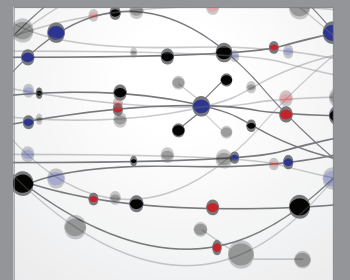

The Scientific World Journal
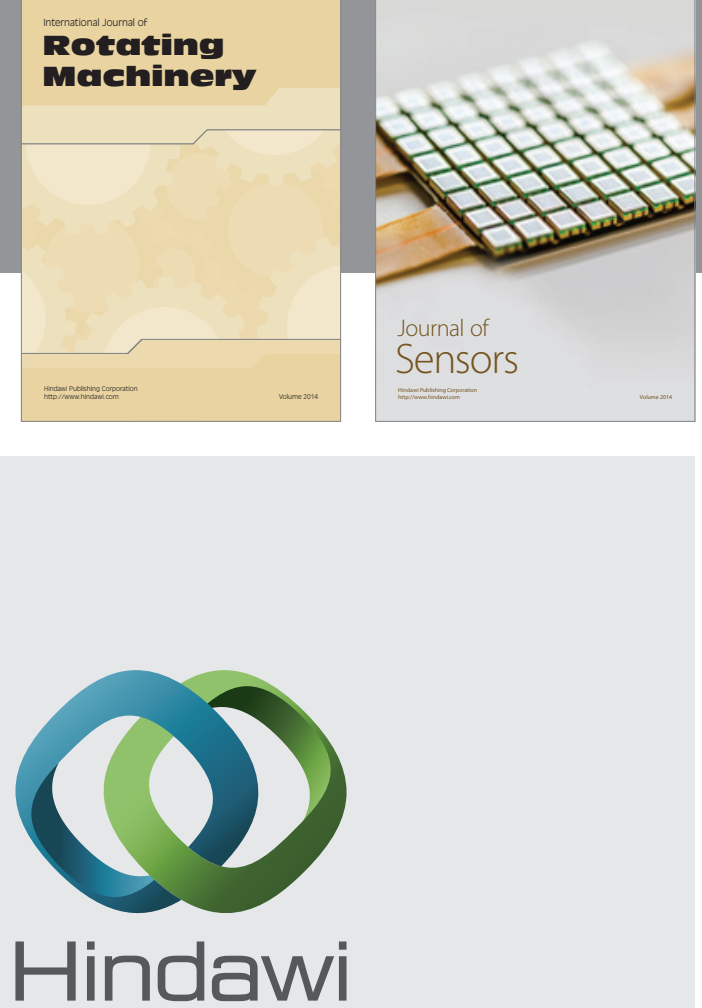

Submit your manuscripts at http://www.hindawi.com
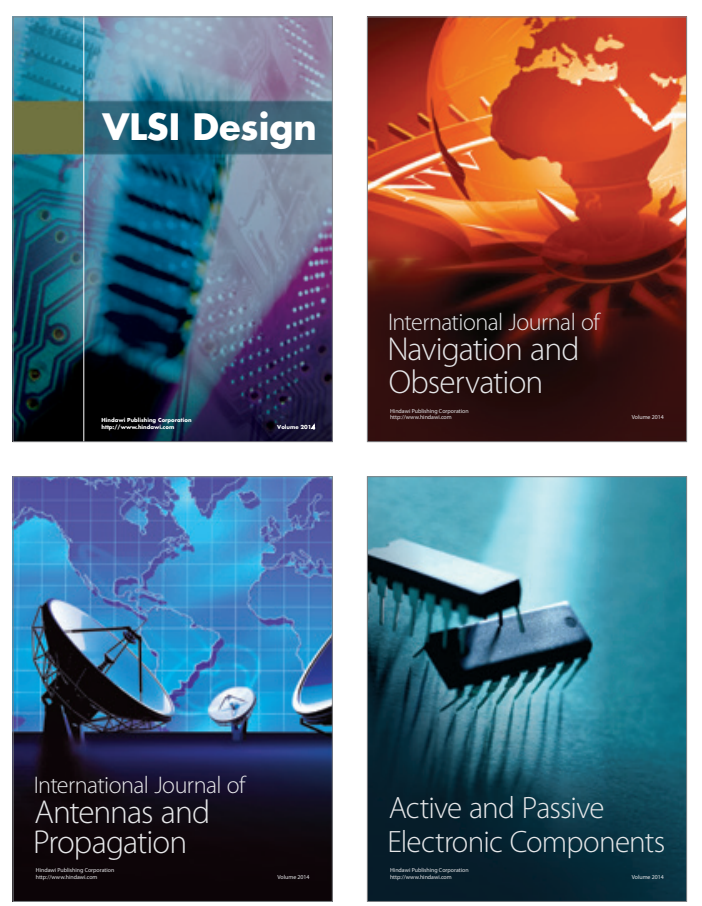
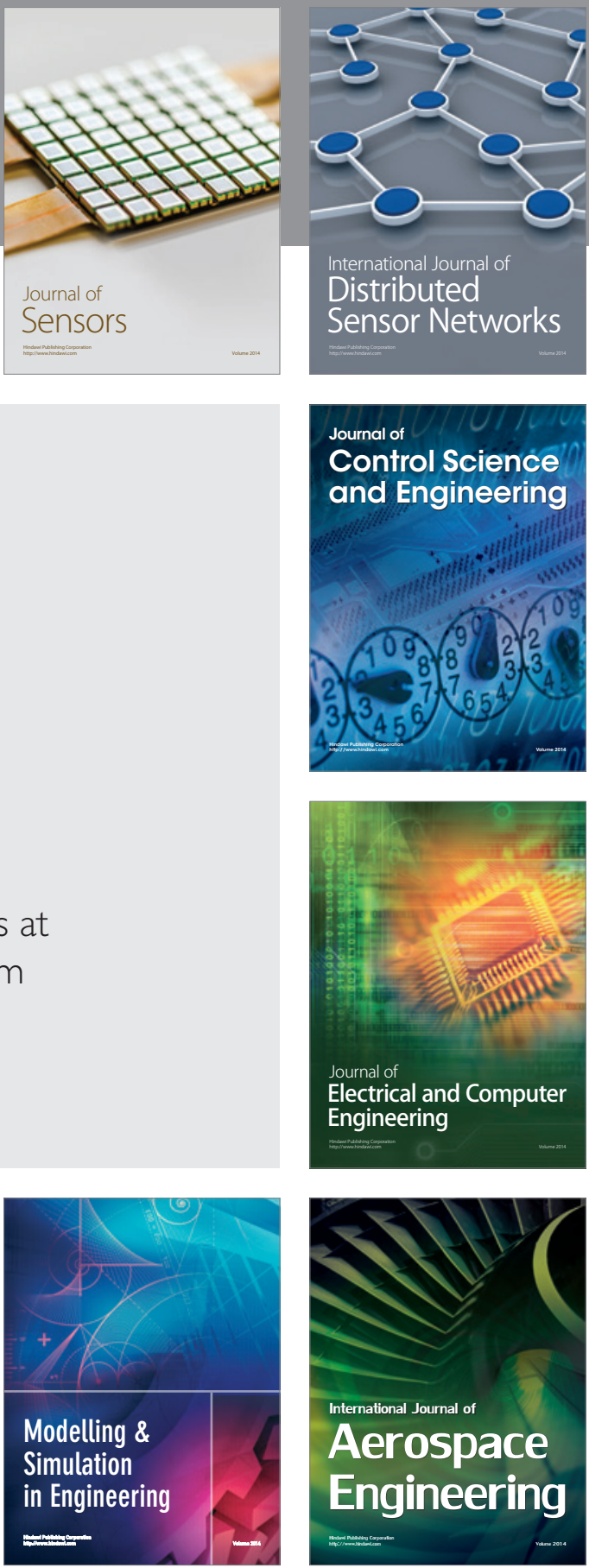

Journal of

Control Science

and Engineering
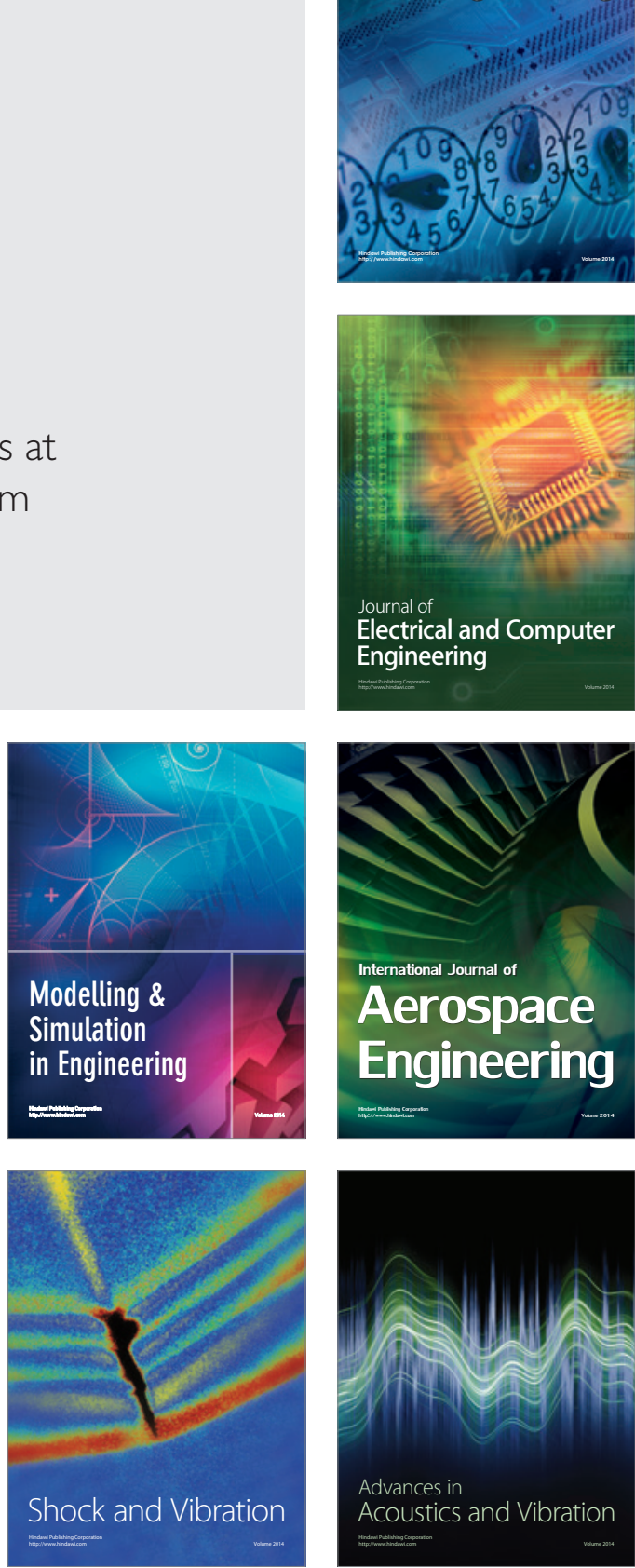